\title{
Optic nerve avulsion, ultrasound and MRI findings
}

\begin{abstract}
Optic nerve avulsion is a rare but serious presentation of ocular trauma. It is characterized by disinsertion of the nerve fibers from the globe at the level of the lamina cribrosa. It is usually associated with a decelerating injury of significant momentum and usually results in a severe and permanent visual loss.1,2 Here we report a patient with optic nerve avulsion secondary to a sporting injury and discuss the role of MRI and B- Scan ultra-sound.
\end{abstract}

Keywords: trauma, optic nerve, avulsion, MRI, imaging, ultrasound, case report
Volume 8 Issue 2 - 2018

\section{Sadik Taju Sherief, Alan John Connor, Cynthia Vanden Hoven, Wai- Ching Lam \\ Department of Ophthalmology and Vision Sciences, University} of Toronto, Canada

\section{Correspondence: Sadik Taju Sherief, Department of \\ Ophthalmology and Vision Sciences, University of Toronto, The Hospital for Sick Children, 555 University Ave, Toronto, ON M5G IX8, Canada, Email goge4000@yahoo.com, sadik. sherief@mail.utoronto.ca}

Received: May 06, 2017 | Published: April 26, 2018

\section{Case report}

A 15-year-old healthy male presented with an ocular injury sustained while playing basketball. He described a finger poke incident with the contact made with the lower aspect of his right eye. Immediately following the injury, he described loss of vision of the right eye. On presentation to the emergency eye clinic 2 hours later, his visual acuity was no light perception in the right eye. A right relative afferent pupillary defect was present. A conjunctival tear and associated chemosis was present in the inferior bulbar conjunctiva. A microhyphaema with $2+$ red cells was found in the anterior chamber but the cornea, lens and intraocular pressure were all normal. Examination of the posterior segment revealed localized vitreous haemorrhage arising from the disc. The disc itself appeared disorganized and recessed behind the globe with an obvious defect in the disc superiorly (Figure 1). Gentle palpation of the globe during fundus examination showed that some streaks of vitreous haemorrhage adjacent to the disc were mobile and could pass through the disc defect with gentle palpation and return into view on release of palpation. There was retinal pallor affecting the superior temporal and nasal aspects of the retina (Figure 2). A B-Scan ultrasound demonstrated alteration in the normal contour of the optic nerve/ globe junction and hypo echogenic area adjacent to the nerve (Figure 3). MRI of the brain and orbit showed disruption in the area of lamina cribrosa of the right eye (Figure 4). At 3 weeks following the injury, progressive dense vitreous haemorrhage prevented visualization of the posterior pole (Figure 5). Subsequent follow up after one month showed that the vitreous haemorhage disperse spontaneously, with persistent optic nerve disorganization and no improvement in vision.

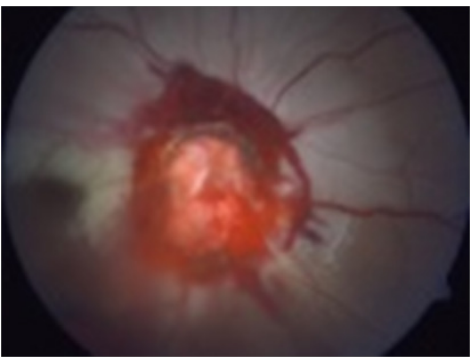

Figure I Fundus picture showing Optic Nerve Avulsion.

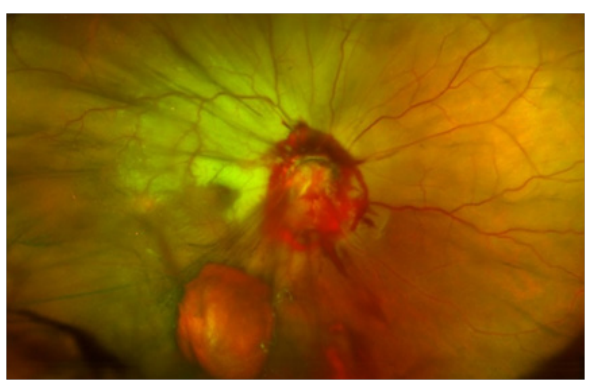

Figure 2 Wide field fundus image demonstrating superior retinal pallor secondary to optic nerve pallor.

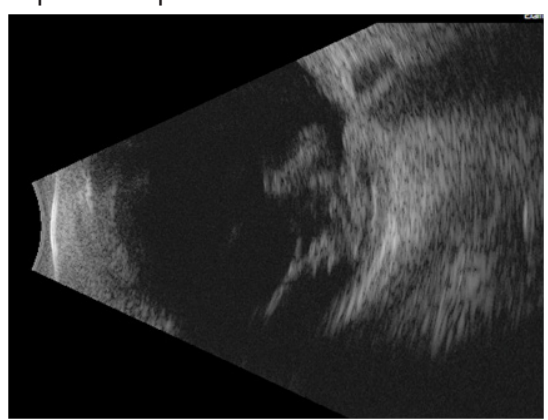

Figure 3 B scan ultrasound demonstrating vitreous haemorrhage, altered optic nerve/globe junction and hypoechogeneic area posterior to globe.

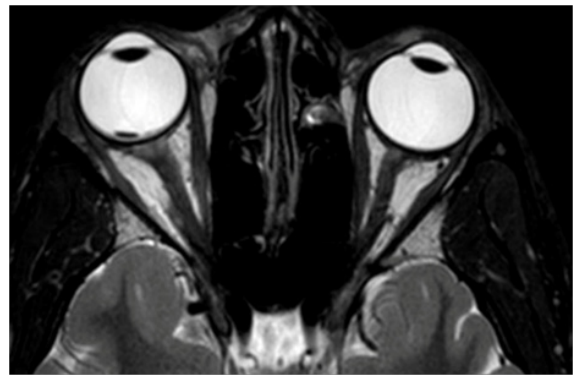

Figure 4 Axial T2 weighted MRI image of orbit demonstrating widened and altered nerve globe junction. 


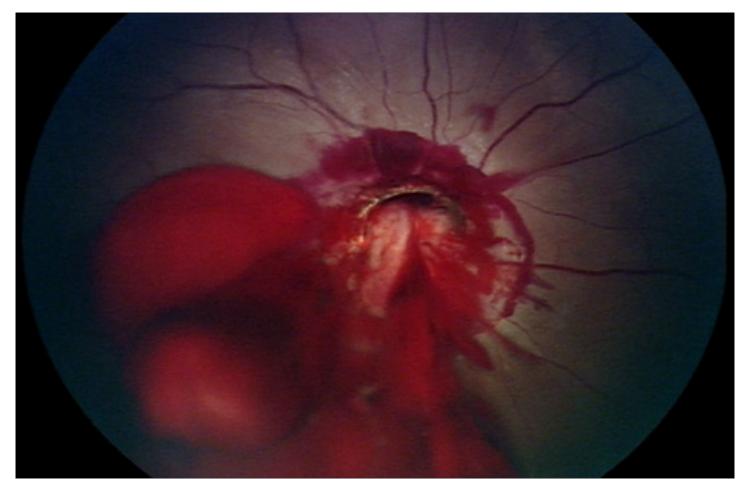

Figure 5 Fundus picture showing vitreous and peripapillary haemorrhage, recessed and disorganized optic nerve.

\section{Discussion}

Optic nerve avulsion (ONA) is uncommon presentation of ocular trauma, where the optic nerve is forcibly disinserted from the retina, choroid and the lamina cribrosa. Both complete and partial avulsions have been described. ${ }^{3,4}$ It typically results from blunt injury to the orbit, concussion of the globe or massive trauma to the face. ${ }^{5}$ Accidents with motor vehicles and bicycles, stick injuries and falls are the common causes of optic nerve avulsion. ${ }^{6,7}$ Several mechanisms have been postulated; sudden extreme rotation of the globe, sudden rise in intraocular pressure leading to the expulsion of nerve out of scleral canal or sudden anterior displacement of the globe. 1, The mechanism of ONA depends on the type of injury. In all cases, sudden forces result in tearing of the optic nerve fibers at the level of the lamina cribrosa. Review suggests the most common mechanism of injury is a severe rotation of the eye leading to rupture of the optic nerve fibers and anterior displacement of the globe. ${ }^{8}$

When the direct trauma, like a finger or pointed object entering the globe, the front of the globe may be rotated toward the object and pushed forward. 2 The torsional and stretching forces on the nerve may result in tearing of the optic nerve fibers, usually starting at the area of the nerve opposite from the location of the entry of the object. ${ }^{9,10}$ In our patient, a finger entering the inferior aspect of the orbit, resulted a partial avulsion of the superior aspect of the optic nerve. The lack of retinal pallor in the inferior retina is suggestive of preservation of the inferior retinal arterioles, if not the nerve fibres themselves were intact.

The diagnosis of ONA is most often made clinically. If the media is clear, the disc is seen to be replaced partially or totally by a hole with variable amounts of surrounding retinal hemorrhages. Direct visualization of the optic nerve may not be possible due to vitreous haemorrhage, in which case ultrasound and MRI imaging can aid the diagnosis. MRI scanning may not be sensitive enough to detect all cases of avulsion. 3, 4 B- scan ultrasonography has also been reported to aid in the diagnosis of ONA. Simsek et al. ${ }^{11}$ reported that B- Scan U/S detected ONA clearly, whereas Foster et al 3 reported B-Scan U/S evaluation failed to detect ONA in 4 cases. So far to the best of our knowledge, eight cases of ONA secondary to basketball injury have been reported. And 7 of the cases were due to finger injury while one patient was secondary to basketball. Similar to our case there was no discerptions of protective goggles. Including our patient, five of them had superior partial avulsion. Except one case, all of the cases were reported to have vitreous hemorrhage. ${ }^{1,2,12-15}$

\section{Conclusion}

Even partial avulsion of the optic nerve can cause severe loss of vision. Given the clinical findings of the anterior segment of the eye and location of ONA in our case, sudden extreme rotation of the globe is likely the mechanism of ONA in cases due to basketball injury with finger poke. This case report showed the helpful role of MRI and BScan ultrasound in detecting ONA.

\section{Acknowledgements}

None.

\section{Conflict of interest}

The author declares that there is no conflict of interest.

\section{References}

1. Sanborn GE, Gonder JR, Goldberg RE, et al. Avulsion of the optic nerve: a clinicopathological study. Can J Ophthalmol. 1984;19:10-16.

2. Park JH, Frenkel M, Dobbie JG, et al. Evulsion of the optic nerve. Am J Ophthalmol. 1971;72:969-971.

3. Williams DF, Williams GA, Abrams GW, et al. Evulsion of the retina associated with optic nerve evulsion. AMJ Ophthalmol. 1987:104:5-9.

4. Foster BS, March GA, Lucarelli MJ, et al. Optic nerve avulsion. Arch Ophthalmol. 1997;115:623-630.

5. Anand S, Harvey R, Sandramouli S. Accidental self-inflicted optic nerve head avulsion. Eye. 2003;17(5):646-647.

6. Steinsapir KD, Goldberg RA. Traumatic optic neuropathy. Surv Ophthalmol. 1994;38(6):487-518.

7. Buchwald HJ, Otte P, Lang GE. Avulsion of the optic nerve following blunt bulbar trauma. Case report and review of the literature. Klin Monatsbl Augenheilkd. 2003;220:303-308.

8. Buchwald HJ, Spraul CW, Wagner P, et al. Optic nerve evulsion: Metaanalysis. Klin Monatsbl Augenheikd. 2001;218(10):635-644.

9. Hykin PG, Gardner ID, Wheatcroft SM. Optic nerve avulsion due to forced rotation of the globe by a snooker cue. Br J Ophthalmol. 1990;74(8):499501.

10. Sawhney R, Kochhar S, Gupta R, et al. Traumatic optic nerve avulsion: Role of ultrasonography. Eye. 2003;17:667-670.

11. Imsek T, Simsek E, Ilhan B, et al. Traumatic optic nerve avulsion. J Pediatr Ophthalmol Strabismus. 2006;43:367-369.

12. Chow AY, Goldberg ME, Frenkel M. Evulsion of the optic nerve in association with basketball injuries. Am J Ophthalmol. 1984;16(1):35-37.

13. Chang M, Eifring DE. Optic nerve Avulsion. Arch Ophthalmol. 1987;105(3):322-323.

14. Roberts SP, Schaumberg DA, Thompson P. Traumatic avulsion of the optic nerve. Optom Vis Sci. 1992;69(9):721-727.

15. Fiedman SM. Optic nerve avulsion secondary to a basketball injury. Ophththalmic Surg Lasers. 1999;30(8):676-677. 